\title{
Sensitivity Analysis for Active Matched Antennas With Non-Foster Elements
}

\author{
Fernando Albarracín-Vargas, Eduardo Ugarte-Muñoz, Vicente González-Posadas, and \\ Daniel Segovia-Vargas
}

\begin{abstract}
During the last years many researchers have been working on the active matching or on non-Foster matching networks for one- and two-port electrically small antennas (ESAs). A new parameter on the sensitivity of the two-port electrically small antenna when loaded with a non-Foster network is presented. This sensitivity analysis will allow us to choose what kind of antennas can be properly matched with non-Foster networks and their position in order to optimize the performance of the design. Then, a typical high $Q$ two-port antenna will be harder to match over a broad bandwidth, since $\left|\mathbf{s}_{21}\right|$ is very small and only agrees with $\left|s_{11}\right|$ over very small frequency bands, yielding very large sensitivity values. However, for these two-port antennas, if high levels of coupling can be engineered for a high $Q$ multiple-port antenna, the return and insertion losses can be similar over larger bandwidths and, hence, the sensitivity can be kept low over larger bandwidths, enabling broader impedance matched bandwidths to be achieved, even for highly resonant antennas.
\end{abstract}

Index Terms-Metamaterials, non-Foster reactance, small-antennas, stability.

\section{INTRODUCTION}

$\mathbf{N}$ ON-FOSTER or active matching of electrically small-antennas (ESAs) has become a very active topic during the last years. Passive matching is limited by the constraints imposed by the Bode [1], Fano [2], and Youla [3] gain-bandwidth criterion. It states that one can achieve a given gain in a very small bandwidth by matching the antenna with lossless capacitors and inductors, or achieve larger bandwidths by including losses in the matching network. This results in a poor efficiency and, hence, a low gain [4]. On the other hand, Wheeler [5], Chu [6], and Thal [7] derived the upper bound for the bandwidth of an ESA, operating at a single-mode resonance, which is strongly limited by the electrical size of the antenna and is inversely proportional to the radiation efficiency [8], [9]. To overcome those constraints, it has been tried to use active matching in order to compensate the natural reactance of the antenna [10], [11] not only at one single frequency but over larger bandwidths by using the negative slope property of the reactance in non-Foster elements. These non-Foster elements (negative capacitors or inductors) are implemented using 2-port active circuits called negative impedance converters (NICs) [12]. A NIC is a 2-port active circuit, in which one port presents the negated version of the impedance loading the other port, so non-Foster elements can be implemented as

$$
Z_{N I C}=-K \cdot Z_{L}
$$

This kind of non-Foster circuits typically presents stability problems that must also be taken into account during the design stage [13], [14] in order to achieve a suitable performance. When integrating one of these circuits with other conventional ones the stability problems become more severe since these external circuits load the NIC and become an indistinguishable part of it. This happens when loading the NIC with electrically small antennas, ESA [15].

It is not until recently that non-Foster forms have been integrated with antenna for active matching networks [16]-[20]. In [16], a microstrip slotted monopole is loaded with a non-Foster form to compensate the antenna reactance and reach broadband matching. In [17], an electrically small caged box loop antenna is loaded with a NIC electrically shielded from the antenna. In [18] and [19], considerations of modeling components for a NIC topology are defined and applied to a parasitic element that aims to modify the distribution of the near-field of the antenna to get broadband matching. Similarly, in [20] a parasitic SRR loaded with a NIC was used to achieve broadband matching. The use of the two-port antennas approach has been proposed in [21] and it seems a suitable design strategy. In addition, a one-port antenna using internal matching network can also be considered as two-port antenna where the second port represents the non-Foster circuit.

In this sense, the current work aims at explaining and analytically finding a suitable location for the non-Foster circuit in the antenna. In addition, it is also seen the effects on the performance of the antenna when changing that position. This paper introduces a sensitivity parameter that shows the effect on the input impedance of the matched antenna versus the variations of loading non-Foster network. This new parameter has been shown to be critical during the design procedure. Finally, in addition to the sensitivity analysis, the type of non-Foster form is also analyzed to take into account the efficiency and its effect on the radiation pattern.

The paper is organized as follows. Section I provides the introduction. Section II presents the aforementioned new sensitivity parameter to analyze the concept of active matching for 


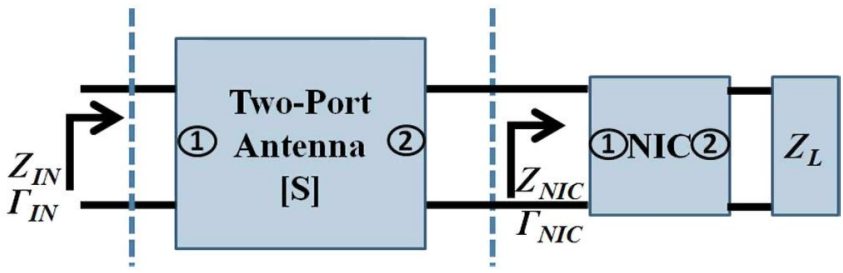

Fig. 1. Active matching of two-port antenna.

two-port antennas; this analysis is critical in order to have a reliable method to show the performance of this kind of antennas. Section III shows the application of the previous parameter in the design of active matching two-port ESA. Three design examples are presented in order to show whether which kind of antennas are suitable to be matched with non-Foster matching networks or not. Finally, the conclusions of the work are given in Section IV.

\section{Active Matching of Two-Port Antennas}

The basic scheme of the active matching for two-port antennas is shown in Fig. 1. It consists of loading one port of the antenna with a non-Foster impedance, $Z_{N I C}$ (implemented by the use of a negative impedance converter, NIC), in such a way that the input impedance at the other port equals some reference impedance $Z_{0}$ in order to obtain larger bandwidths.

The reflection coefficient of this loaded two-port network at its input, $\Gamma_{I N}$, is given by (2a). As the goal is to have a matched antenna, the input reflection coefficient should be zero over its intended design bandwidth. Then, once ( $2 \mathrm{a}$ ) is set to zero, the analytic or optimum reflection coefficient, in terms of impedance matching, that the NIC must provide, $\Gamma_{N I C}^{a n}$, can be extracted as $(2 b)$

$$
\begin{aligned}
\Gamma_{I N} & =S_{11}+\frac{S_{12} \cdot S_{21} \cdot \Gamma_{N I C}}{1-S_{22} \cdot \Gamma_{N I C}}=0 \\
\rightarrow \Gamma_{N I C}^{a n} & =\frac{S_{11}}{S_{22} \cdot S_{11}-S_{12} \cdot S_{21}} .
\end{aligned}
$$

Therefore, the non-Foster analytic impedance, $Z_{N I C}^{a n}$, needed to match the antenna at port 1 can be expressed in terms of the two-port $\boldsymbol{S}$-parameters of the antenna as

$$
Z_{N I C}^{a n}=Z_{0} \cdot\left(\frac{S_{22} \cdot S_{11}-S_{12} \cdot S_{21}+S_{11}}{S_{22} \cdot S_{11}-S_{12} \cdot S_{21}-S_{11}}\right) .
$$

When this impedance $Z_{N I C}^{a n}$ is placed at port 2, the reflection coefficient at port $1, \Gamma_{I N}$, would ideally equal 0 at all frequencies and, hence, large bandwidths can be obtained.

However, from a practical point of view, due to the fact that the NIC impedance is quite sensitive to variations on its load impedance [12], [13], it is also very important to analyze a new parameter such as the sensitivity of this solution, Sens, which is given by

$$
\Delta \Gamma_{I N}=\underbrace{\left.\frac{\partial \Gamma_{I N}}{\partial \Gamma_{N I C}}\right|_{\Gamma_{N I C}=\Gamma_{N I C}^{a n}}}_{\text {Sensitivity:Sens }} \cdot \Delta \Gamma_{N I C}=\text { Sens } \cdot \Delta \Gamma_{N I C} .
$$

This sensitivity parameter provides information about how potential variations in the impedance of the NIC $\left(\Gamma_{N I C}\right)$ vs. the analytic one given by (2b) affects the input reflection coefficient, $\Gamma_{I N}$, and the obtained impedance (or VSWR) bandwidth $B W_{V}$ of the two-port antenna (see Fig. 1). Under a constraint of a maximum VSWR criterion (e.g., $V S W R_{\max }=1.92$ for $\left|S_{11}\right|=$ $-10 \mathrm{~dB}$ ) we can obtain a minimum value for the magnitude of $\Gamma_{I N}$, in (5); (e.g., $\left|\Gamma_{I N_{-} \min }\right|=0.316$ for $\left|S_{11}\right|=-10 \mathrm{~dB}$ ) and relates its changes with the changes in VSWR

$$
V S W R_{\max }=\frac{1+\left|\Gamma_{I N_{-} \min }\right|}{1-\left|\Gamma_{I N_{-} \min }\right|}
$$

In (6), the impedance bandwidth $B W_{V}$ is defined as the frequency interval around a frequency $\omega_{0}$ at which the VSWR equals $V S W R_{\max }$ [22]. $Q$ is the quality factor of the antenna. In [23], expressions similar to (6) are derived for planar antennas with linear and circular polarizations

$$
B W_{V} \approx \frac{2 \cdot \omega_{0}\left(V S W R_{\max }-1\right)}{Q \cdot \sqrt{V S R W_{\max }}} .
$$

An expression to relate $B W_{V}$ with the Sens parameter and changes in the $\left|\Gamma_{N I C}\right|$ can be derived as follows:

$$
\begin{aligned}
& \left.\frac{\partial B W_{V}}{\partial \Gamma_{N I C}}\right|_{\substack{\Gamma_{N I C}=\Gamma_{N I C}^{a n} \\
\left|\Gamma_{I N}\right|=\left|\Gamma_{I N-\min }\right| \\
V S W R=V S W R_{\max }}}=\frac{\partial B W_{V}}{\partial V S W R} \cdot \frac{\partial V S W R}{\partial\left|\Gamma_{I N}\right|} \cdot \frac{\partial\left|\Gamma_{I N}\right|}{\partial \Gamma_{N I C}} \\
& \Delta B W_{V} \approx \frac{8 \omega_{0}}{Q} \cdot \text { Sens } \cdot \Delta \Gamma_{N I C} .
\end{aligned}
$$

High values of the sensitivity (Sens) imply that very small changes in $\Gamma_{N I C}$ will result in very large changes in $\left|\Gamma_{I N}\right|$ and, hence, in the antenna impedance bandwidth $B W_{V}$.

For a two-port antenna the sensitivity Sens can be easily obtained by applying (4) into (2a). The result is given in (9) and (10) so it can be seen how the sensitivity only depends on the $S$-parameters of the two-port antenna. For these reasons, the placement of the two ports must be studied in order to reach practical realizable designs. The two ports must be placed in such a way that the sensitivity is minimized. Otherwise, any small change in the impedance provided by the NIC will dramatically affect the antenna performance in terms of the impedance bandwidth

$$
\begin{aligned}
\text { Sens } & =\left.\left|\frac{S_{21}+S_{12}}{\left(1-S_{12} \Gamma_{N I C}\right)^{2}}\right|\right|_{\Gamma_{N I C}=\Gamma_{N I C}^{a n}} \\
& =\left|\frac{\left(S_{11} S_{22}-S_{21} S_{12}\right)^{2}}{S_{21} S_{12}}\right| .
\end{aligned}
$$

If the two ports are symmetrically placed $\left(S_{11}=S_{22}\right)$, (4) can be rewritten as

Sens $=\left|\frac{\left(S_{11}{ }^{2}-S_{21}{ }^{2}\right)^{2}}{S_{21}{ }^{2}}\right|=\left|\frac{\left(\left(S_{11}+S_{21}\right) \cdot\left(S_{11}+S_{21}\right)\right)^{2}}{S_{21}{ }^{2}}\right|$.

From the previous expressions, it can be concluded that the sensitivity will be smaller when the transmission parameter $\left(S_{21}\right)$ equals, in magnitude, the reflection parameter $\left(S_{11}\right)$. This provides an idea about what kind of antennas are more suitable 
to be used in non-Foster active matched two-port antennas designs, as it will be shown in Section III. The Sens parameter will be approximately flat for non-resonant antennas while will present different transmission peaks for resonant antennas. In general, very resonant antennas will present higher values of Sens and will be harder to match along a broad bandwidth since $\left|S_{21}\right|$ is very small and would only agree with the $S_{11}$ for very small frequency bands. Then, if high levels of coupling can be engineered for a high $\mathrm{Q}$ two-port antenna (or even a multiport one), the return and insertion losses can be similar over larger bandwidths and the sensitivity can be kept low over larger bandwidths, enabling a broadband active matching design. In other words, if $\left|S_{21}\right|$ is low, in comparison with $\left|S_{11}\right|$, the non-Foster element connected to port 2 has not much influence in the port 1 . Expressing it in a different way, in terms of the antenna size, when it decreases in electrical terms (i.e., in an ESA), that is when $k a<1$ where $a$ is the radius of the smallest sphere enclosing the entire antenna system at the resonance frequency $\omega_{0}$, and $k$ is the free-space wavenumber (i.e., $k=\omega_{0} / c$ ), the quality factor $Q$ of the antenna increases dramatically [19]. However, we could find a low sensitivity point over its structure, so a broadband active matched antenna design would be feasible, as we will see in Section III devoted to a loop antenna.

Once the sensitivity has been analyzed and the placement of the two ports has been chosen, an additional constraint on the antenna efficiency has to be considered. If the $Z_{N I C}^{a n}$ presents a real part (resistive part) different from zero, the radiation efficiency would decrease at frequencies far away from the resonant ones because the energy would be mostly dissipated in heat and not radiated. That is an important constraint in active matching design. For that reason, once a low sensitivity value for a certain non-Foster circuit location has been found, a further adjustment on the obtained $Z_{N I C}^{a n}$ with a non-zero real part will be done over the desired frequency range in order to have both broad bandwidth and good efficiency. This will be analyzed in the Section III with the development of the proposed antennas.

Finally, other factors such as the feasibility of $Z_{N I C}^{a n}$ (the number of non-Foster elements needed to implement $Z_{N I C}^{a n}$ and their values) and the stability of the whole design, once you have a two-port stable network [24] (i.e., one non-Foster element), must be taken into account. For the stability purpose, the authors have proposed in [15] the use of the normalized determinant function (NDF) which allows analyzing the stability of the whole design, NIC integrated with the two-port antenna. However, in this paper we will focus on the sensitivity analysis.

\section{DESIGN EXAMPLES}

In this section, we will analyze three different antennas: a small loop-antenna, a conventional rectangular patch and a metamaterial-inspired multi-frequency patch antenna. According to the previous section, it will be shown how the first and the second cases are suitable choices for active matching in terms of sensitivity, while the third one presents an extremely high sensitivity and, hence, should not be recommended for active matching.

\section{A. Small Loop Antenna}

The first antenna under test is a small-loop antenna (i.e., since the factor $k a \leq 1$ for frequencies up to $636 \mathrm{MHz}$ ), with a diam-

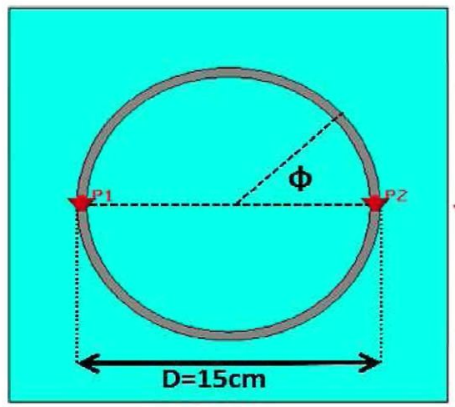

(a)

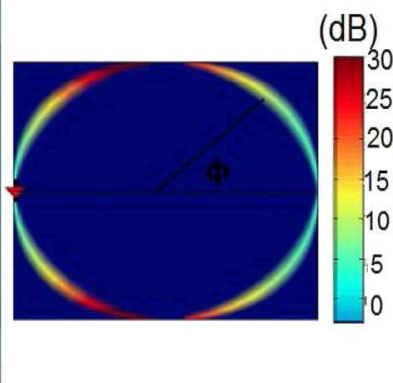

(b)
Fig. 2. Small loop antenna. (a) Sketch of the two-port loop antenna. (b) Sensitivity of the antenna versus port-2 placement.

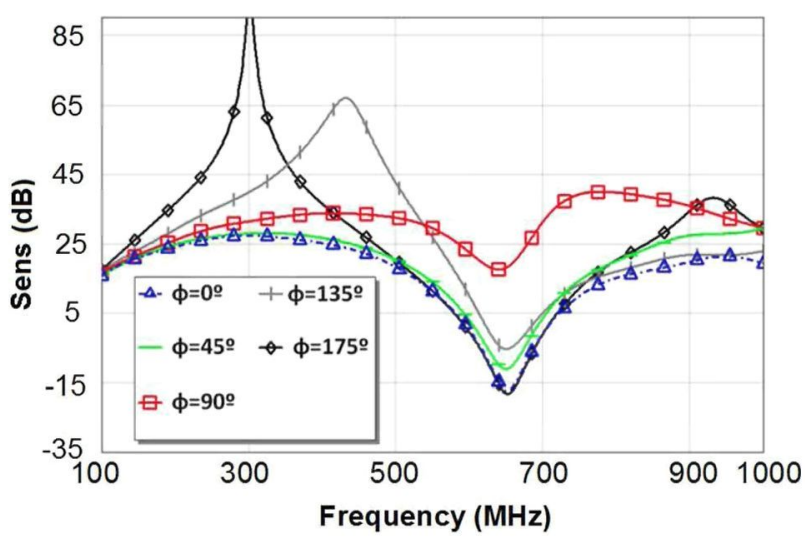

Fig. 3. Parameter Sens VS frequency for different $\Phi$.

eter $\mathrm{D}=15 \mathrm{~cm}$, over a 0.5 -mm-thick substrate with $0.035-\mathrm{mm}$ thick metal layer; the width of the wire is $3 \mathrm{~mm}$ and the substrate relative permittivity is 4.5. As shown in Fig. 2(a), the antenna has two ports. The first one is the feeding port, and the second one is loaded with a non-Foster element. A parametric study has been undertaken by varying the position of the second port (through the angle $\boldsymbol{\Phi}$ ) in order to obtain the sensitivity at each point. Fig. 2(b) shows the averaged parameter Sens at each point. It can be seen how the averaged sensitivity is maximum when $\Phi=90^{\circ}(\overline{\text { Sens }}=30 \mathrm{~dB})$ and minimum when $\Phi$ is around $0^{\circ}$ or $180^{\circ}(\overline{\text { Sens }}=5 \mathrm{~dB})$. Therefore, the non-Foster element should be connected at these positions of lowest sensitivity. It is important to remark that, in Fig. 2(b), the sensitivity has been averaged over the interest bandwidth $350-600 \mathrm{MHz}$.

Fig. 3 shows the variation of the sensitivity with the frequency for different values of $\boldsymbol{\Phi}$. The optimum placement of the nonFoster is for $\Phi=0^{\circ}$ in the proposed bandwidth.

Once the position of the NIC has been chosen according to the sensitivity criterion, a second analysis concerning the efficiency has been undertaken. The $S$ parameters and the real and imaginary parts of $Z_{N I C}^{a n}$ when port 2 is at $\Phi=0^{\circ}$ are depicted in Fig. 4(a) and (b), respectively.

From Fig. 4(b) it can be seen that the real part of the nonFoster network is small, but different from zero, in the desired frequency range. Then, in order not to reduce the antenna efficiency, the analytic impedance $Z_{N I C}^{a n}$ is modified by rejecting its real part and keeping an imaginary part as close as possible to the one of $Z_{N I C}^{a n}$ in a frequency range as large as possible in the 


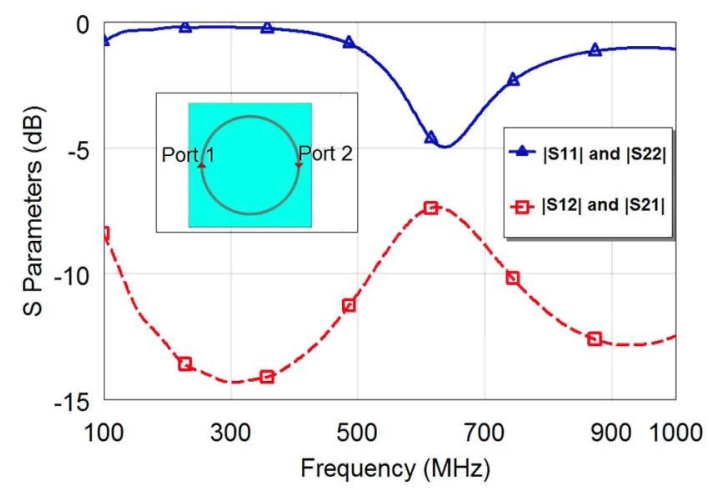

(a)

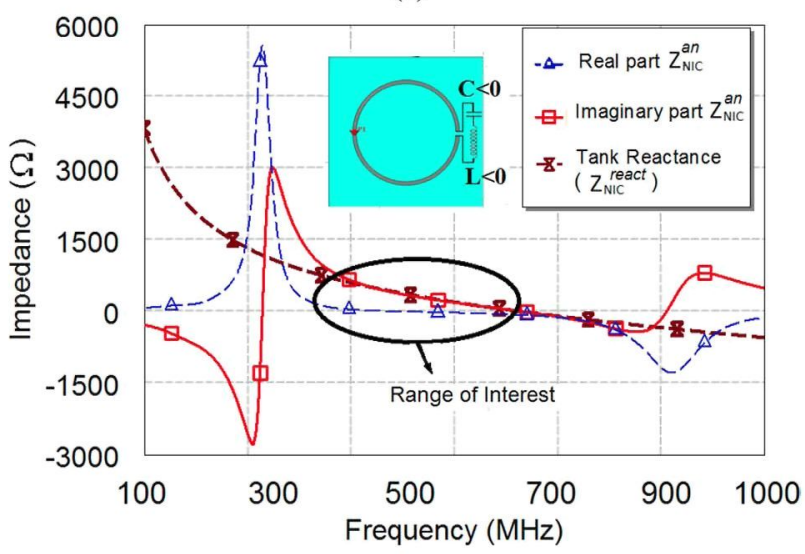

(b)

Fig. 4. (a) Simulated S parameters versus frequency of the two-port small loopantenna. (b) Impedance response of the analytic NIC, $Z_{N I C}^{a r}$ and its approach with a purely reactive series tank $Z_{N I C}^{\text {react }}$ with non-Foster components at the lowest sensitivity point in the antenna.

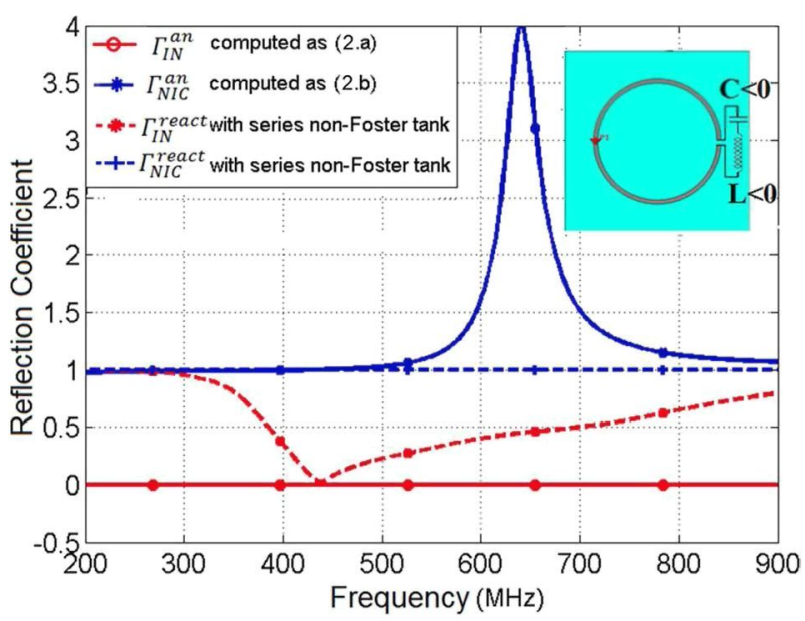

Fig. 5. Reflection coefficient for the analytic and reactive load impedances $\left(\Gamma_{N I C}^{a n}\right.$ and $\left.\Gamma_{N I C}^{r e a c t}\right)$ and their corresponding overall input reflection coefficient for the small-loop antenna $\Gamma_{I N}$ and $\Gamma_{I N}^{\text {react }}$ ).

lowest sensitivity point. This can be achieved with a series reactive tank (suboptimal impedance, $Z_{N I C}^{\text {react }}$ ) $(\mathrm{L}<0$ and $\mathrm{C}<0$ ) that gets a wider bandwidth in the desired frequency range as can be seen in Fig. 5.

In addition to the curves representing $Z_{N I C}^{a n}$ and the tank impedance $Z_{N I C}^{r e a c t}$, it is useful to represent the corresponding

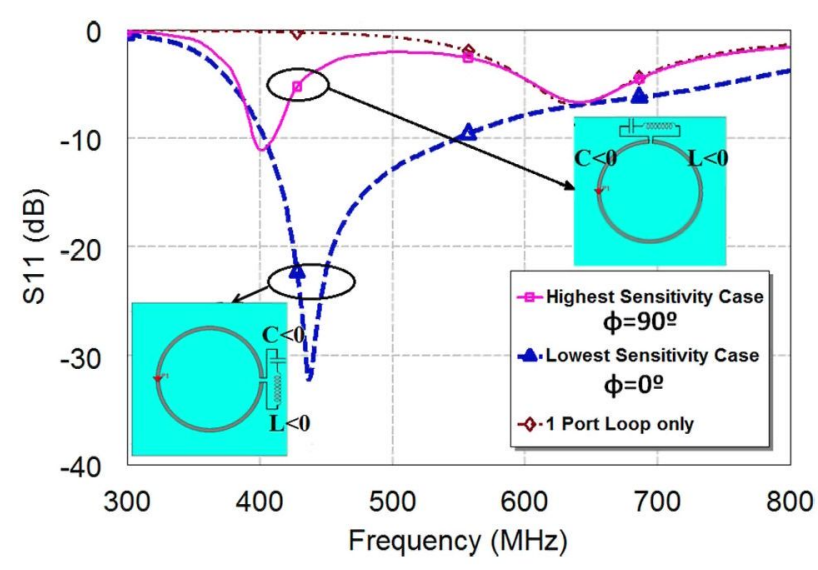

Fig. 6. $S_{11}(\mathrm{~dB})$ of the two designs.

reflection coefficients for the previous load impedances and for the resulting $\Gamma_{I N}$ (see Fig. 1). Then, if the two-port antenna is loaded with the ideal $\Gamma_{N I C}^{a n}$, it can be seen that the corresponding $\Gamma_{I N}$ is always 0 according to (2a). However, when paying attention to $\Gamma_{N I C}^{a n}$, it can be appreciated that after the resonance in Fig. 5 its magnitude is higher than 1 so that it is potentially unstable. Thus, a stability analysis has to be done to avoid that risk. Second, if the $\Gamma_{\text {NIC }}^{a n}$ is replaced with the proposed $\Gamma_{N I C}^{r e a c t}$ it can be seen that its magnitude is 1 in the desired frequency margin (due to its purely reactive components); this yields better performance from the stability point of view. Then, when seeing the input reflection coefficient, $\Gamma_{I N}^{\text {react }}$, it can be seen that broad matching condition can be achieved (as seen in Fig. 6).

On the other hand, when the non-Foster element is placed at $\Phi=90^{\circ}$ it is not possible to match the antenna, neither with purely reactive elements nor with the analytic NIC impedance, due to the high sensitivity. The sensitivity is so high that very small variations (even using ideal elements, as it is the case) in the impedance of the non-Foster element $Z_{N I C}^{a n}$ or $Z_{N I C}^{\text {react }}$ results in very large variations of $\Gamma_{\mathrm{IN}}$ and, hence, in the bandwidth as depicted in Fig. 6.

The results are summarized in Table I: When the port is placed at the lowest sensitivity point $\Phi=0^{\circ}$, the obtained relative bandwidth is $30.7 \%$. On the other hand, when the port is placed at a high sensitivity point $\Phi=90^{\circ}$, the maximum relative bandwidth that can be obtained degrades to values lower than 5\%. With these two very basic examples it has been shown that the sensitivity plays an important role when designing antenna active matching networks based on non-Foster circuits. Then, the NIC must be connected at low sensitivity points in order to obtain feasible and robust designs.

Finally, one comment should be done concerning the current distribution along the loop with the NIC in order to check if the radiation pattern is affected by the inclusion of the NIC. The analysis that has been done to see the current distribution when the proposed tank impedance $Z_{N I C}^{\text {react }}$ is inserted at $\Phi=0^{\circ}$ and at $\Phi=90^{\circ}$ is shown in Fig. 7(a). From that figure it can be seen that, at a frequency in the range of interest, there is no change in the net current distribution along the loop when the NIC is inserted in comparison when no NIC is inserted. In this way, no 
TABLE I

Simulated RETURN LOSSES.

\begin{tabular}{lcc}
\hline \hline \multicolumn{1}{c}{ Antenna } & $\begin{array}{c}\text { Absolute }(-10 \mathrm{~dB}) \\
\text { Bandwidth } \\
(\mathrm{MHz})^{\mathrm{a}}\end{array}$ & Relative Bandwidth \\
\hline $\begin{array}{l}\text { Lowest Sensitivity } \\
\text { Case }\left(\Phi=0^{\circ}\right)\end{array}$ & 146 & $30.7 \%$ \\
$\begin{array}{l}\text { Highest Sensitivity } \\
\text { Case }\left(\Phi=90^{\circ}\right)\end{array}$ & 15 & $3.8 \%$ \\
\hline \hline
\end{tabular}

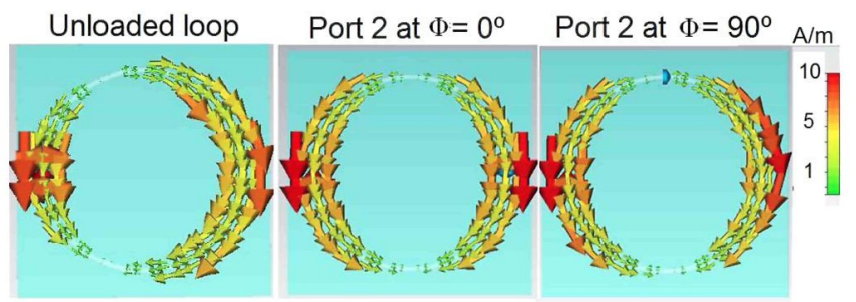

(a.)

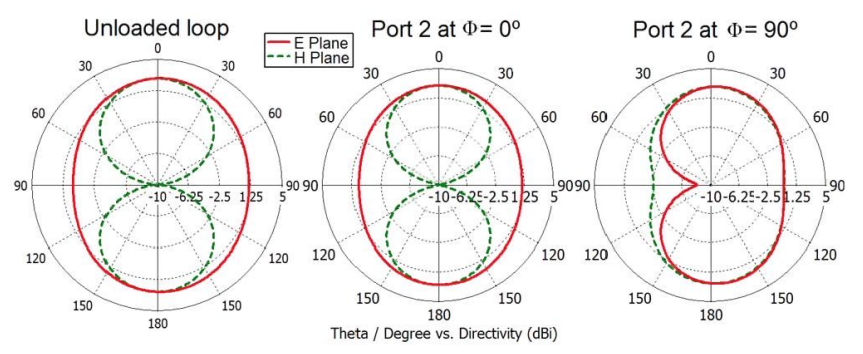

(b.)

Fig. 7. (a) Current distribution along the loop and (b) simulated radiation pattern when the NIC $\left(Z_{N I C}^{\text {react }}\right)$ is placed at $\Phi=0^{\circ}$ and $\Phi=90^{\circ}$, respectively, at $500 \mathrm{MHz}$

change occurs in the radiation pattern as can be seen in Fig. 7(b).

\section{B. Conventional Patch Antenna}

For a two-port conventional patch antenna working at 300 $\mathrm{MHz}$, as the one depicted in Fig. 8 over an FR4 $\left(\varepsilon_{\mathrm{r}}=4.35\right)$ substrate of $40-\mathrm{mm}$ width; a parametric study has been performed in order to evaluate the averaged sensitivity $\overline{\operatorname{Sens}}$ (i.e., averaged over an bandwidth of interest chosen from $150 \mathrm{MHz}$ to $300 \mathrm{MHz}$ ) as a function of the position of the two ports. Fig. 8 shows the results when the port 1 (referred to a $Z_{0}=50 \Omega$ ) is placed at $\{x=-85 \mathrm{~mm}, y=0 \mathrm{~mm}\}$ and port 2 moves along the patch.

It can be seen how the averaged sensitivity presents desired and low values (blue color) when port 2 is placed at positions bounded by $x=\{90 \mathrm{~mm}, \ldots, 30 \mathrm{~mm}\}$ and $y \approx 0 \mathrm{~mm}$. For this situation both ports excite non-orthogonal modes so the coupling, $S_{21}$ or $S_{12}$, is large and more similar to $S_{11}$ and $S_{22}$. Hence, the denominator of the second part in (9) is larger while the numerator is lower so the sensitivity is minimized. When the ports are placed in other region the sensitivity is very large (red colors). The largest sensitivity is obtained when the ports are placed in an orthogonal way. In this case, the ports excite orthogonal modes being the coupling $\left(S_{21}\right)$ very small and very

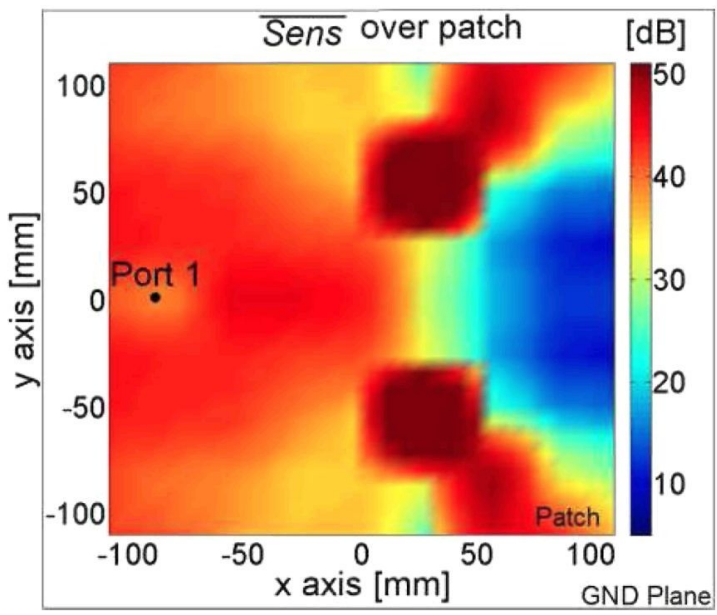

Fig. 8. Averaged sensitivity $\overline{\text { Sens }}(\mathrm{dB})$ of the patch antenna versus port-2 placement.

different from $S_{11}$ and $S_{22}$. The numerator of (9) is very large while the denominator is very small resulting in huge sensitivities.

Considering the value of $Z_{N I C}^{a n}$ and its feasibility of implementing, the lowest sensitivity point $(\{x=110 \mathrm{~mm}, y=$ $0 \mathrm{~mm}, \overline{\text { Sens }}=4.02 \mathrm{~dB}\}$ ) has to be discarded, because of the need of three non-Foster elements to realize $Z_{N I C}^{a n}$ [see Fig. 9(a) with a series combination of a negative inductor with a negative parallel LC tank]. Another possible design is studied for the point $\{x=27 \mathrm{~mm}, y=0 \mathrm{~mm}, \overline{\text { Sens }}=30 \mathrm{~dB}$. Fig. 9(b) shows the $\mathrm{S}$ parameters for the two-port antenna with this port placing. Fig. 9(c) shows the analytic impedance, $Z_{N I C}^{a n}$ and the approximation obtained using ideal non-Foster elements. The obtained return losses are shown in Fig. 9(d). It can be seen that the most feasible design is when the port is placed at $x=27 \mathrm{~mm}$ since it only needs a negative parallel $L C$ tank with two non-Foster elements instead of three of them to realize $Z_{N I C}^{r e a c t}$ It is also important to remark that the sensitivity Sens depends on the frequency as the $S$-parameters do as shown in Fig. 9(b) the magnitude of the sensitivity parameter Sens over the desired bandwidth when the port 2 is located at $x=27 \mathrm{~mm}$, $y=0 \mathrm{~mm}$.

The planar nature of the patch antenna structure requires an analysis of the current distribution after including the non-Foster network at port 2 to see if the active matching modifies the radiation pattern. Fig. 10(a) shows a change in the current distribution at $200 \mathrm{MHz}$ for the conventional patch antenna when is loaded with $Z_{N I C}^{\text {react }}$. It can be seen that for the loaded case there is a maximum of current in the middle of the patch what leads to the monopolar like radiation pattern seen in Fig. 10(b), different from the one expected in a frequency away from resonance one for a conventional patch antenna (i.e., a magnetic dipole like pattern).

\section{Multi-Frequency Patch Antenna Based on Metamaterials}

The third antenna under study is a patch antenna loaded with a $3 \times 3$ array of mushrooms metamaterial particles, as it is shown in Figs. 11(a) and 11(b) [25]. The antenna is fed through two coupled ports. As in the previous case, one of the ports is used to 


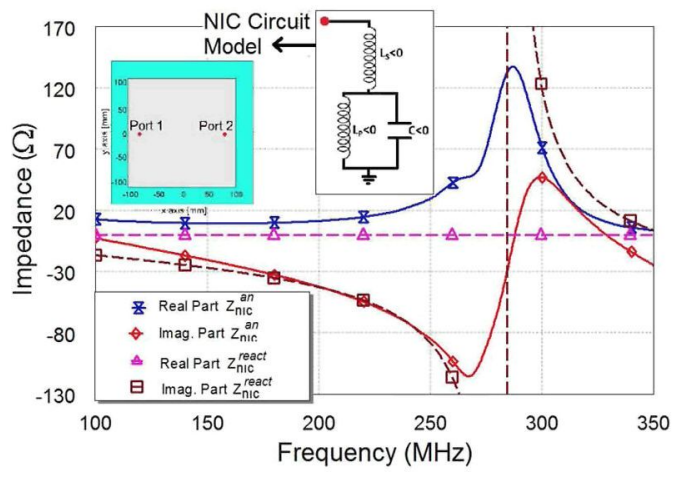

(a)

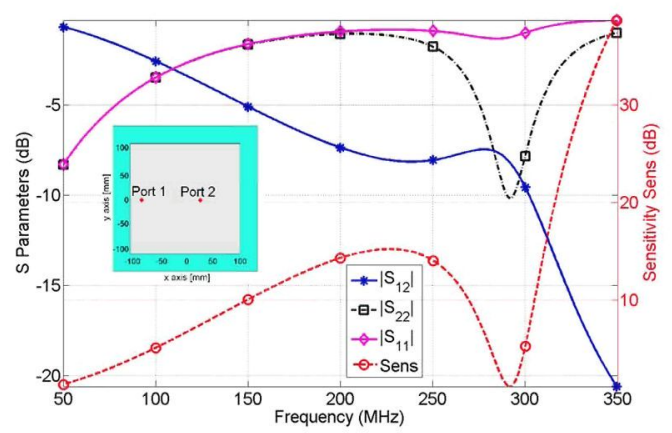

(b)

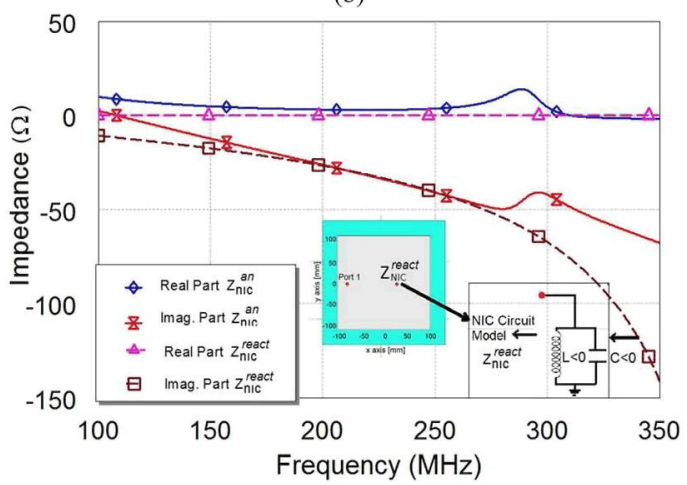

(c)

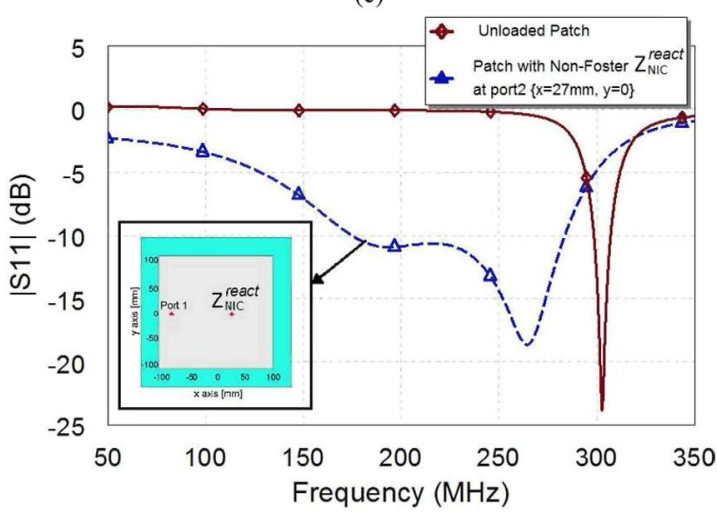

(d.)

Fig. 9. (a) $Z_{N I C}^{a n}$ and ideal non-Foster approximation for $Z_{N I C}^{\text {react }}$ at $\{x=$ $100 \mathrm{~mm}$ and $y=0 \mathrm{~mm}$. (b) Sensitivity Sens and $S$ parameters versus frequency for port 2 located at $\{x=27 \mathrm{~mm}$ and $y=0 \mathrm{~mm}\}$. (c) $Z_{N I C}^{a n}$ and an ideal non-Foster approximation for $Z_{N I C}^{r e a c t}$ at $\{x=27 \mathrm{~mm}$ and $y=0 \mathrm{~mm}\}$. (d) $\left|S_{11}\right|$ for the unloaded and 2-port patch with $Z_{\text {NIC }}^{\text {react }}$.

feed the antenna, while the other one is loaded with a non-Foster element in order to try to improve its bandwidth.

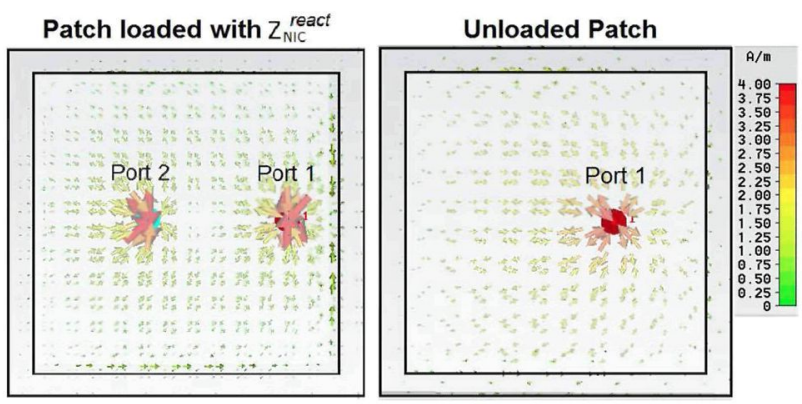

(a.)

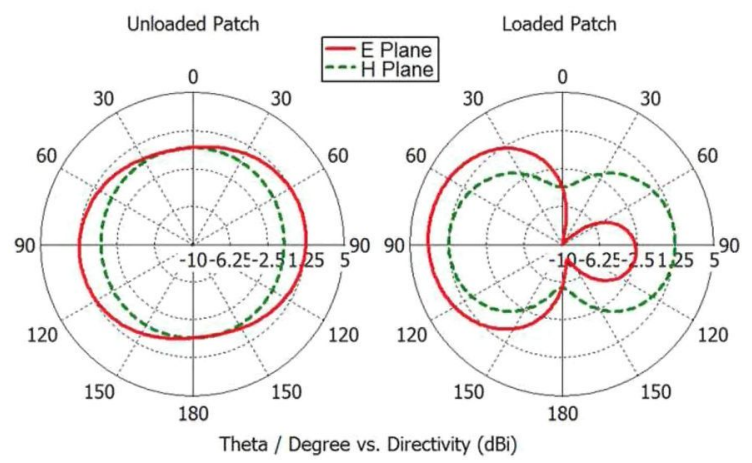

(b.)

Fig. 10. (a) Current distribution at $200 \mathrm{MHz}$ for the conventional patch antenna and (b) the simulated radiation patterns for the unloaded and the loaded with $Z_{N I C}^{\text {react }}$ patch, respectively, with a very small ground plane

The equivalent transmission line (TL) model of the antenna consists of a left-handed (LH) section between two right-handed (RH) sections, as shown in Fig. 11(a). The propagation constant in the LH section $\beta^{\mathrm{LH}}$ is negative so, according to the resonant condition given by (11), it is possible to obtain additional modes to the fundamental one

$$
\beta_{n} L=\beta_{n}^{R H} d+\beta_{n}^{L H} \ell=k_{1} f_{n} d-\frac{k_{2}}{f_{n}} \ell=n \pi .
$$

This antenna has been chosen since the miniaturization degree that can be achieved is important [25]. This mode is inherently narrowband, as it is provided by the metamaterial structures [26] so, as in the previous cases, it will be studied if it is possible to improve the bandwidth by using active matching. The antenna is designed to work in the $\mathbf{- 1}$ mode in the $150-\mathrm{MHz}$ band. The patch is a square one, $25 \mathrm{~cm}$ side, the side of the mushroom is $18.3 \mathrm{~cm}$, the gap between mushrooms is $1 \mathrm{~mm}$, the diameter of the vias is $1 \mathrm{~mm}$ and, finally, the antenna is designed on a 5-cm-high substrate with unity permittivity.

First, the sensitivity parameter is studied in order to see whether this antenna is suitable to be used with non-Foster forms. Then, the sensitivity has been computed for different placements of the second port. An average value of the sensitivity in the desired bandwidth (140 MHz-170 MHz) has been obtained. The results of this parametric analysis are shown in Fig. 12. It can be seen how the sensitivity is extremely large for all the possible positions of the second port.

The S-parameters of the two- port antenna can help us to understand why the sensitivity is so large for this antenna. Fig. 13 


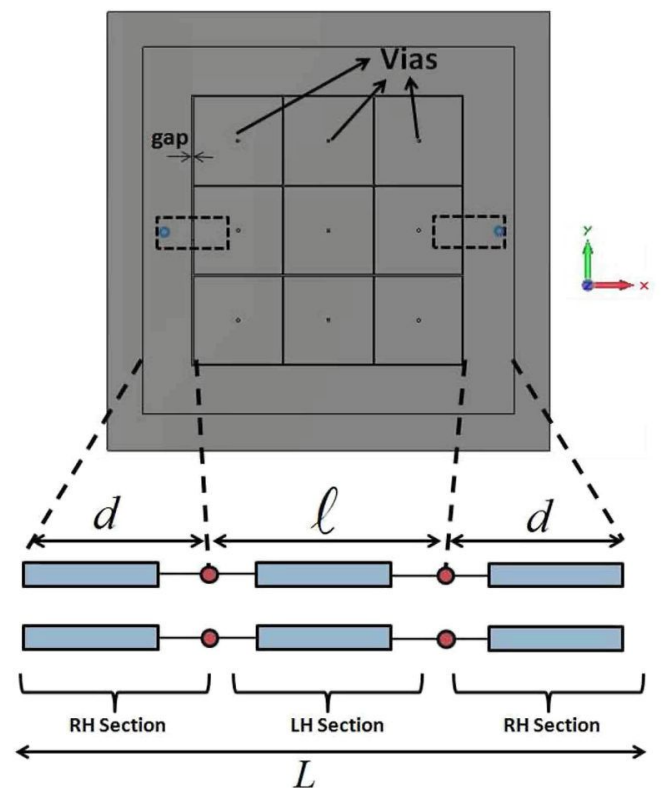

(a)

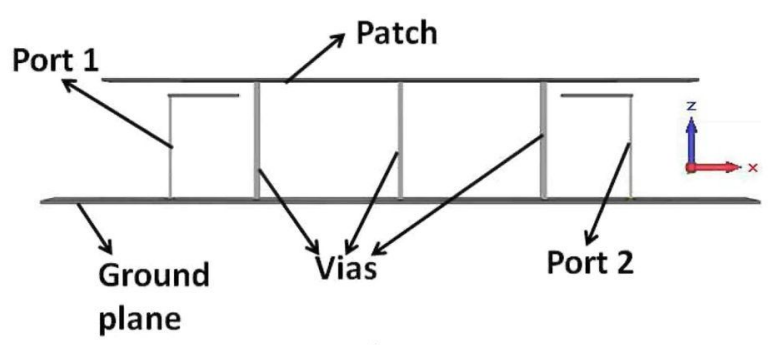

(b)

Fig. 11. Multifrequency antenna based on metamaterials. (a) Top-view and (b) lateral view. $L=25 \mathrm{~cm} \times 25 \mathrm{~cm}, l=18.3 \mathrm{~cm} \times 18.3 \mathrm{~cm} . h=5 \mathrm{~cm}$.

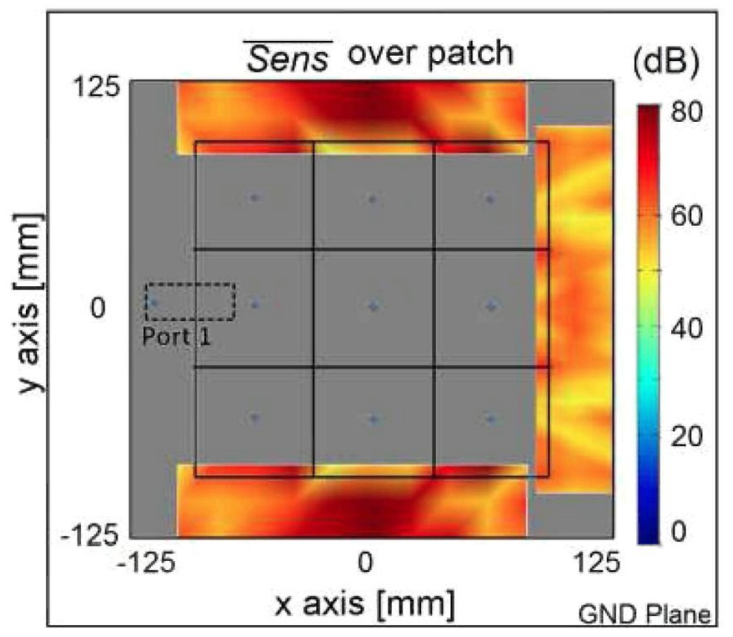

Fig. 12. Averaged sensitivity $\overline{\text { Sens }}$ (dB) of the antenna versus port-2 placement.

shows the S-parameters as well as the computed sensitivity using (9). From that figure, it can be seen that the coupling between the two-ports is very low. When this happens, $\boldsymbol{S}_{21}$ is very low and the sensitivity is very large according to (9). For this reason the sensitivity is only relatively small at very narrow frequency bands where the $S_{21}$ is larger: $144 \mathrm{MHz}, 151 \mathrm{MHz}$, and $156 \mathrm{MHz}$. For this reason, this antenna, or any antenna with

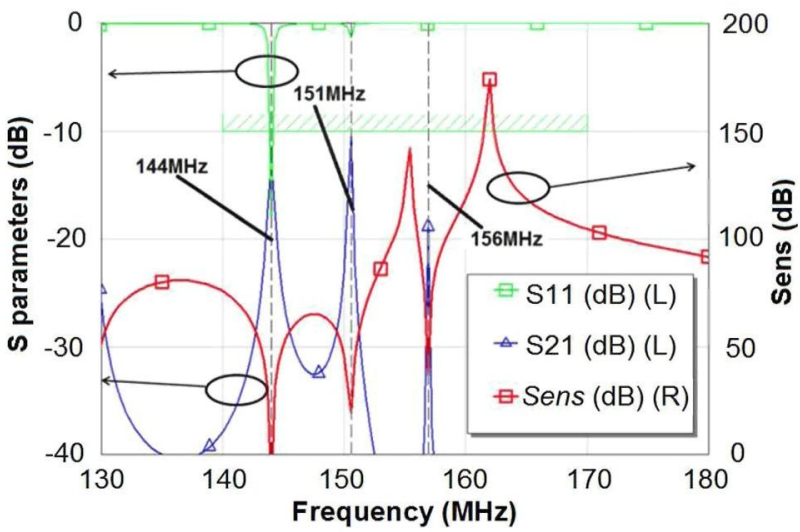

Fig. 13. $S$-parameters and sensitivity Sens of the patch loaded with metamaterial forms.

similar response, cannot be used in two-port active matching designs.

In order to obtain low sensitivities and thus, feasible designs, the antenna should present large $S_{21}$ values, which is logical, because if $S_{21}$ were very small, the influence at port 1 of the non-Foster element connected at port 2 will be also very small [see (9) and (10)].

As aforementioned, very resonant antennas such as the patch antenna loaded with metamaterials presented in this section are not appropriate, since $S_{21}$ is very small and only agrees with the $S_{11}$ for very small frequency bands. It should be emphasized that the quality factor is not directly proportional to the sensitivity. Then, for any proposed two-port antenna, a high quality factor $Q$ does not necessary imply a high sensitivity value for a certain port distribution over the antenna and a low quality factor does not necessary imply a low sensitivity factor. In spite of the $Q$ value over the frequency interval of interest the sensitivity will have to be studied to find out the best position where the NIC can be included. As the value of the sensitivity is not suitable, the integration of non-Foster forms in this two-port antenna will not be possible and larger bandwidths will not be achieved.

Although from the sensitivity analysis it has already been concluded that it is not possible to use this antenna for the integration with a non-Foster network, a final analy sis on the current distribution and on the corresponding radiation pattern is also presented. Fig. 14(a) shows the comparison between the simulated current distribution at $144 \mathrm{MHz}$ when the patch is loaded with $Z_{N I C}^{\text {react }}$ at the symmetrically located port 2 (with respect to port 1) and the unloaded patch. It can be seen that for the case of loading with the non-Foster element (reactive case) there is a maximum of current in the middle of the patch what leads to a monopolar radiation pattern instead of the typical dipolar one associated to the metamaterial patch with no loading seen in Fig. 14(b).

Finally, it can be concluded that nonresonant antennas such as the small loop and conventional patch antenna presented in Sections III-A and III-B, respectively, are more appropriate. These antennas present similar values of $S_{11}$ and $S_{21}$ over larger bandwidths so that the sensitivity is also kept low over larger bandwidths, making broadband designs possible without degrading the antenna efficiency if the real part of $Z_{N I C}^{a n}$ has low 


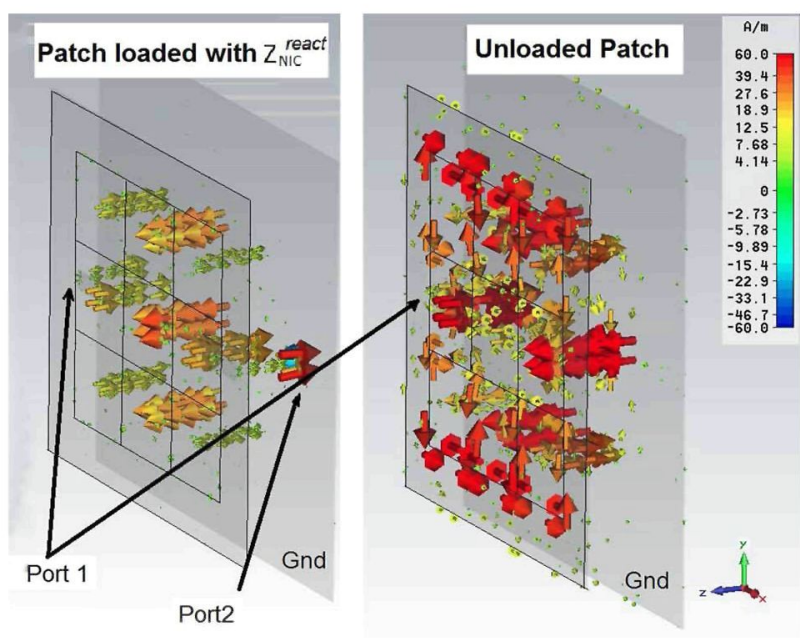

(a.)

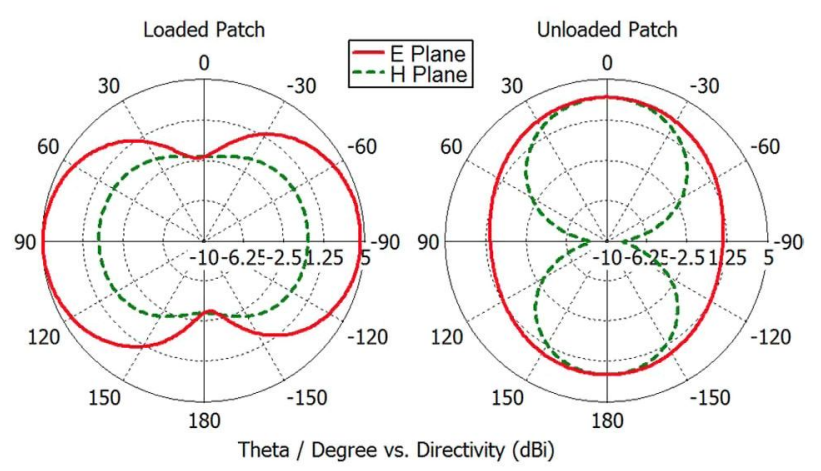

(b.)

Fig. 14. (a) Current distribution in both the loaded with $Z_{N I C}^{\text {react }}$ and the unloaded metamaterial patch and (b) the simulated radiation patterns for both cases (with a very small ground plane).

values over the frequency range of interest. Then, a design procedure for this kind of non-Foster forms can be proposed as follows.

1) Study the sensitivity parameter in order to see whether the proposed antenna presents suitable points to integrate a non-Foster form. In case so you can go ahead with the following points (this happens, for instance, for the loop and for the conventional patch but not for the metamaterial patch).

2) Choose a suitable NIC that does not greatly affect the efficiency of the antenna. For that reason the impedance chosen is a suboptimal one with reactive performance.

3) Study the current distribution in order to see that the radiation modes are the same as in the original antenna (this happens for the loop but not for conventional patch or the patch loaded with metamaterial particles).

\section{Conclusion}

It has been shown that the sensitivity Sens is a very important design parameter when working with active matching of two-port antennas. The introduction of the sensitivity parameter allows us to see the dependence on the input impedance with variations on the analytic NIC impedance. The ports must be placed at points of low sensitivity. In this way, small variations of the non-Foster element, (variations in the NICs) will not degrade the matching performance, so once a low sensitivity location is chosen, the design must be made over a frequency range where the real part of $Z_{N I C}^{a n}$ has low values in order to not to degrade the antenna efficiency. If the ports are placed in such a way that high sensitivities are obtained, the design will not work properly, since any small variation in the implemented non-Foster element (which are unavoidable in practice) will yield in high variations in the input impedance, and hence in the bandwidth.

Moreover, the feasibility must be also taken into account; because in some placements the analytic impedance needed (specifically the optimum reactance) is very difficult to achieve using practical NICs. For this reason, a tradeoff between sensitivity and feasibility should be studied for each particular case.

\section{ACKNOWLEDGEMENTS}

The authors would like to acknowledge the support of the COST IC1102 Project.

\section{REFERENCES}

[1] H. W. Bode, Network Analysis and Feedback Amplifier Design. New York, NY, USA: D. Van Nostrand, 1947.

[2] R. M. Fano, "Theoretical limitations on the broadband matching of arbitrary impedances," J. Franklin Inst., vol. 249, no. 1, pp. 57-83, 1950.

[3] D. C. Youla, "A new theory of broad-band matching," IEEE Trans. Circuit Theory, vol. CT-11, no. 1, pp. 30-50, Mar. 1964.

[4] E. Sussman-Fort and R. M. Rudish, "Non-Foster impedance matching of electrically-small antennas," IEEE Trans. Antennas Propag., vol. 57, no. 8, Aug. 2009.

[5] H. A. Wheeler, "Fundamental limitations of small antennas," Proc. IRE, vol. 35 , no. 12 , pp. 1479-1484, Dec. 1947.

[6] L. J. Chu, "Physical limitations of omni-directional antennas," J. Appl. Phys., vol. 19, no. 12, pp. 1163-117, Dec. 1948.

[7] H. L. Thal, "New radiation Q limits for spherical wire antennas," IEEE Trans. Antennas Propag., vol. 54, no. 10, pp. 2757-2763, Oct. 2006.

[8] S. R. Best, "Low Q electrically small linear and elliptical polarized spherical dipole antennas," IEEE Trans. Antennas Propag., vol. 53, no. 3, pp. 1047-1053, Mar. 2005.

[9] J. S. McLean, "A re-examination of the fundamental limits on the radiation Q of electrically small antennas," IEEE Trans. Antennas Propag., vol. 44, no. 5, pp. 672-676, May 1996.

[10] R. M. Foster, "A reactance theorem," Bell Syst. Tech. J., vol. 3, pp. 259-267, 1924.

[11] W. Geyi, P. Jarmuszewski, and Y. Qi, "The Foster reactance theorem for antennas and radiation Q," IEEE Trans. Antennas Propag., vol. 48, no. 3, Mar. 2012.

[12] J. G. Linvill, "Transistor negative impedance converters," Proc. IRE, vol. 46, no. 6, Jun. 1993.

[13] E. Ugarte-Muñoz, A. Kiricenko, S. Hrabar, and D. Segovia-Vargas, "Stability of non-Foster reactive elements for use in active metamaterials and antennas," IEEE Trans. Antennas Propag., vol. 60, no. 7, Jul. 2012.

[14] S. D. Stearns, "Circuit stability theory for non-Foster circuits," in IEEE MTT-S Int. Microw. Symp. Dig., 2013, pp. 1-3.

[15] E. Ugarte-Muñoz, D. Segovia-Vargas, V. Gonzalez-Posadas, and J. L. Jiménez-Posadas, "Non-Foster matching of electrically small antennas. Stability considerations," in Proc. Int. Symp. Antennas and Propagation (APSURSI), Jul. 2012, pp. 1-2.

[16] H. Mirzaei and G. V. Eleftheriades, "A resonant printed monopole antenna with an embedded non-Foster matching network," IEEE Trans. Antennas Propag., vol. 61, no. 11, pp. 5363-5371, Nov. 2013.

[17] J. Church, J. Chie, L. Xu, J. D. Rockway, and D. Arceo, "UHF electrically small box cage loop antenna with a embedded non-Foster load," Antennas Wireless Propag. Lett., 2014.

[18] N. Zhu and R. Ziolkowski, "Active metamaterial-inspired broad-bandwidth efficient, electrically small antennas," IEEE Antennas Wireless Propag. Lett., vol. 10, 2011.

[19] N. Zhu and R. Ziolkowski, "Broad-bandwidth, electrically small antenna augmented with an internal non-Foster element," IEEE Antennas Wireless Propag. Lett., vol. 11, pp. 1116-1120, 2012. 
[20] M. Babuto, A. Monti, F. Bilotti, and A. Toscano, "Design of a non-Foster actively loaded SRR and application in metamaterial inspired components," IEEE Trans. Antennas Propag., vol. 61, no. 3, pp. 1219-1226, Mar. 2013

[21] S. Koulouridis, "Non-Foster circuitry design for antennas," in Proc. 5 th Eur. Conf. Antennas and Propagation, Apr. 2011, pp. 237-239.

[22] A. D. Yaghiian and S. R. Best, "Impedance, bandwidth, and Q of antennas," IEEE Trans. Antennas Propag., vol. 53, no. 4, pp. 1298-1324, Apr. 2005.

[23] W. L. Langston and D. R. Jackson, "Impedance, axial-ratio, and receive-power bandwidths of microstrip antennas," IEEE Trans. Antennas Propag., vol. 52, no. 10, pp. 2269-2273, Oct. 2004.

[24] A. Platzker and W. Struble, "Instabilities diagnosis and the role of K in microwave circuits," in IEEE MTT-S Int. Microw. Symp. Dig., Jun. 1993 , pp. 1185-1189.

[25] F. J. Herraiz-Martinez, V. González-Posadas, L. E. García-Muñoz, and D. Segovia-Vargas, "Multifrequency and dual-mode patch antennas partially filled with left-handed structures," IEEE Trans. Antennas Propag., vol. 56, no. 8, pp. 2527-2539, Aug. 2008.

[26] S. Hrabar, I. Krois, and A. Kiricenko, "Towards active dispersionless ENZ metamaterial for cloaking applications," Metamaterials, vol. 4 no. $2-3$, pp. $89-97$. 